\title{
Logic, Probability and Learning, or an Introduction to Statistical Relational Learning
}

\author{
Luc De Raedt \\ Department of Computer Science, Katholieke Universiteit Leuven \\ Celestijnenlaan 200 A B-3001, Heverlee, Belgium \\ luc.deraedt@cs.kuleuven. be
}

Probabilistic inductive logic programming (PILP), sometimes also called statistical relational learning, addresses one of the central questions of artificial intelligence: the integration of probabilistic reasoning with first order logic representations and machine learning. A rich variety of different formalisms and learning techniques have been developed and they are being applied on applications in network analysis, robotics, bio-informatics, intelligent agents, etc. This tutorial starts with an introduction to probabilistic representations and machine learning, and then continues with an overview of the state-of-the-art in statistical relational learning. We start from classical settings for logic learning (or inductive logic programming) namely learning from entailment, learning from interpretations, and learning from proofs, and show how they can be extended with probabilistic methods. While doing so, we review state-of-the-art statistical relational learning approaches and show how they fit the discussed learning settings for probabilistic inductive logic programming.

This tutorial is based on joint work with Dr. Kristian Kersting.

\section{References}

1. De Raedt, L.: Logical and Relational Learning. Springer, Heidelberg (in press, 2008)

2. De Raedt, L.: Kristian Kersting: Probabilistic logic learning. SIGKDD Explorations 5(1), 31-48 (2003)

3. De Raedt, L., Kersting, K.: Probabilistic Inductive Logic Programming. In: BenDavid, S., Case, J., Maruoka, A. (eds.) ALT 2004. LNCS (LNAI), vol. 3244, pp. 19-36. Springer, Heidelberg (2004) 